\title{
Complexidade Parametrizada de Cliques e Conjuntos Independentes em Grafos Prismas Complementares
}

\author{
Priscila Camargo, Alan D. A. Carneiro, Uéverton S. Santos \\ ${ }^{1}$ Programa de Pós-Graduação em Computação - IC UFF, Niterói, Brasil \\ \{pcamargo, aaurelio, usouza\}@ic.uff.br
}

\begin{abstract}
Resumo. Um grafo do tipo prisma complementar $G \bar{G}$ surge da união disjunta do grafo $G$ e do seu complementar $\bar{G}$ e a adição de um emparelhamento perfeito entre os vértices correspondentes em $G$ e $\bar{G}$. Os problemas clássicos determinar se no grafo existe uma clique de ordem $k$ e determinar se no grafo existe um conjunto independente de ordem $k$ são provados NP-completos quando o grafo de entrada é um prisma complementar. Neste trabalho, estudamos a complexidade de ambos os problemas em grafos prismas complementares sob a ótica da complexidade parametrizada. Nós provamos que estes problemas possuem um núcleo e, portanto são Tratáveis por Parâmetro Fixo (FPT). Em seguida, mostramos que ambos não admitem núcleo polinomial.
\end{abstract}

\begin{abstract}
The complementary prism $G \bar{G}$ arises from the disjoint union of the graph $G$ and its complement $\bar{G}$ by adding the edges of a perfect matching joining pairs of corresponding vertices of $G$ and $\bar{G}$. The classical problems of graph theory, clique and independent set were proved NP-complete when the input graph is a complemantary prism. In this work, we study the complexity of both problems in complementary prisms graphs from the parameterized complexity point of view. First, we prove that these problems have a kernel and therefore are Fixed-Parameter Tractable (FPT). Then, we show that both problems do not admit polynomial kernel.
\end{abstract}

\section{Introdução}

Fazendo uma alusão ao conhecido prisma, um prisma complementar é composto por duas bases: um grafo $G$ e o seu complementar $\bar{G}$. A união destas bases é dada através de um emparelhamento perfeito entre os vértices correspondentes em $G$ e $\bar{G}$. Os prismas complementares foram introduzidos na última década por [Haynes et al. 2007]. Em [Duarte et al. 2017] foram analisadas as complexidade de cliques, conjuntos independentes, $k$-dominação e $P_{3}$-convexidade em prismas complementares. Em [Duarte et al. 2017] foi provado que tanto o problema da clique quanto o problema dos conjuntos independentes são NP-completos para esta classe de grafos.

Neste artigo, investigamos os problemas das cliques e dos conjuntos independentes em grafos prismas complementares sob a ótica da complexidade parametrizada. Devido a restrições de espaço, todas as provas serão omitidas.

\section{Definições e Notações}

Nesta seção serão apresentados os principais conceitos e notações utilizados neste trabalho. 
Grafos: Utilizamos as notações e conceitos padrões de teoria dos grafos e quaisquer notações ou conceitos não definidos podem ser encontrados em [Bondy and Murty 1976]. Grafo $G$ é um par ordenado $(V(G), E(G))$, onde $V(G)$ é um conjunto finito de vértices e $E(G)$ um conjunto de arestas formadas por pares de vértices. Denotaremos por $\bar{G}$ o complemento de um grafo $G$. $\bar{G}$ é composto pelo mesmo conjunto de vértices de $G$ e pelo conjunto de arestas complementares de $G$. Isto é, se a aresta $u v$ existir em $G$, ela não existirá em $\bar{G}, \operatorname{logo}$ os vértices $u$ e $v$ não serão adjacentes em $\bar{G}$. Entretanto, se os vértices $u$ e $v$ não são adjacentes em $G$, existirá a aresta $u v$ em $\bar{G}$. O número de vértices de um grafo $G$ é dito ser a ordem ou cardinalidade de $G$. Um subgrafo de um grafo $G$ é um grafo $H$ tal que $V(H) \subseteq V(G)$ e $E(H) \subseteq E(G)$, denotado por $H \subseteq G$. Um subgrafo $H$ é um subgrafo induzido de $G$ por um subconjunto $X \subseteq V(G)$ (representado por $H[X]$ ) se, para qualquer par de vértices $x$ e $y$ de $H, x y$ é uma aresta de $H$ se e somente se $x y$ for uma aresta de $G$. Clique é um subgrafo induzido completo, isto é, cada par de vértices desse subgrafo é conectado por uma aresta. Denotaremos por $k$-CLIQUE o problema de encontrar uma clique de tamanho $k \mathrm{em}$ um grafo $G$. Conjunto independente de um grafo $G$ é um subconjunto $S$ de vértices de $G$ tal que em $S$ não existem dois vértices adjacentes. Denotaremos por $k$-CONJUNTO INDEPENDENTE o problema de encontrar um conjunto independente de tamanho $k$ em um grafo $G$. Grafos split são grafos cujo conjunto de vértices pode ser particionado em 2 subconjuntos distintos: um conjunto independente e uma clique. Denotamos como $k$-SPLIT o problema de determinar se um grafo $G$ possui um subgrafo split $H$. Denotaremos por $k$-RAMSEY o problema de encontrar uma clique de tamanho $k$ ou um conjunto independente de tamanho $k$ em um grafo $G$. Tal problema não admite núcleo polinomial a menos que $N P \subseteq \operatorname{coNP} /$ poly.

Complexidade Parametrizada: As definições e conceitos básicos de complexidade parametrizada podem ser encontrados em [Cygan et al. 2015]. Um problema parametrizado $\Pi(k)$ é chamado Tratável por Parâmetro Fixo (Fixed Parameter Tratable (FPT)) se existe um algoritmo $A$ (chamado algoritmo FPT) que computa corretamente qualquer instância $I=(\chi, k)$ onde $\chi$ denota o tamanho da entrada e decide se $I$ é uma instância sim ou não em tempo $f(k) \cdot|\chi|^{O(1)}$ para alguma função computável $f$. Dessa maneira, se $k$ é definido com um valor pequeno, o crescimento da função em relação a $\chi$ é pequeno.

A kernelização ou redução a um núcleo é uma poderosa técnica comumente utilizada na obtenção de algoritmos FPT para problemas parametrizados. A técnica consiste em reduzir uma instância $I$ parametrizada a uma nova instância equivalente $I^{\prime}$ tal que $I^{\prime}$ de alguma forma é limitada pelo parâmetro $k$. A instância reduzida $I^{\prime}$ é chamada de kernel ou núcleo. O núcleo de um problema parametrizado é formalmente definido a seguir:

Definição 1. Núcleo: Seja $\Pi(k)$ um problema parametrizado. $\Pi(k)$ possui um núcleo se para toda instância $I=(\chi, k)$ existe um algoritmo A executável em tempo polinomial que fornece como saída uma instância equivalente $I^{\prime}=\left(\chi, k^{\prime}\right)$ tal que $k^{\prime} \leq g(k)$ para alguma função computável $g$.

O ponto chave na técnica de redução a um núcleo é o tamanho do núcleo em relação ao parâmetro onde busca-se encontrar o menor núcleo já que possibilita o desenvolvimento de um algoritmo FPT mais eficiente. Para demonstrar o limite inferior no tamanho do kernel, usamos uma Transformação Polinomial Parametrizada, chamada PPT-redução. Tal redução é definida a seguir.

Definição 2. PPT-redução: Seja $\Pi(k)$ e $\Pi^{\prime}\left(k^{\prime}\right)$ dois problemas parametrizados onde 
$k^{\prime} \leq g(k)$ para alguma função polinomial $g: \mathbb{N} \rightarrow \mathbb{N}$. Uma PPT-redução de $\Pi(k)$ a $\Pi^{\prime}\left(k^{\prime}\right)$ é uma redução $R$ tal que:

(i) Para toda instância $I=(\chi, k)$, temos que $I \in \Pi(k)$ se e somente se $R(I) \in$ $\Pi^{\prime}\left(k^{\prime}\right)$;

(ii) $R$ é computável em tempo polinomial em relação a $k$.

\section{Resultados}

Nesta seção provamos que tanto $k$-CLIQUE quanto $k$-CONJUNTO INDEPENDENTE são FPT's e não admitem núcleo de tamanho polinomial.

\subsection{Tratabilidade Parametrizada}

Afim de provar que $k$-CLIQUE e $k$-CONJUNTO INDEPENDENTE são FPT quando o grafo de entrada é $G \bar{G}$ e o parâmetro é o tamanho da solução $k$, utilizamos o conhecido limite superior do número de Ramsey (Teorema 1). No Teorema de Ramsey, dados inteiros positivos $k$ e $l$ existe um inteiro positivo $R=r(k, l)$ tal que, em qualquer grafo com $R$ de vértices, sempre existe uma clique de tamanho $k$ ou conjunto independente de tamanho $l$.

Teorema 1. [Erdös and Szekeres 1935] Para todos inteiros positivos $k$ e l, vale que: $r(k, l) \leq\left(\begin{array}{c}k+l-2 \\ k-1\end{array}\right)$.

Teorema 2. $k$-Clique $e$-COnJunto IndePEndente são FPT quando o grafo de entrada é $G \bar{G}$ e o parâmetro é o tamanho da solução $k$.

O Teorema 2, portanto, apresenta um núcleo para o problema $k$-CLIQUE ou $k$ CONJUNTO INDEPENDENTE quando o grafo de entrada é um prisma complementar. Dessa forma, ambos os problemas são solucionáveis em tempo $f(k) \cdot n^{O(1)}$ e pertencem à classe FPT.

\subsection{Limite Inferior de Redução a um Núcleo}

Em complexidade parametrizada, um problema $\Pi(k)$ pertence a classe FPT se e somente se $\Pi(k)$ possuir um núcleo. Em geral esses núcleos obtidos possuem tamanhos exponenciais, ou até pior, em relação ao parâmetro. Dessa forma, é natural para qualquer problema FPT questionarmos quão bom é o núcleo, ou mesmo se é possível a obtenção de um núcleo menor. Em geral busca-se um núcleo de tamanho polinomial, embora existam diversos resultados positivos sobre a existência de núcleos polinomiais ou até mesmo lineares. Recentemente apareceram os primeiros resultados a respeito da inviabilidade destes núcleos [dos Santos and dos Santos Souza 2015].

Mostramos que embora $k$-CLIQUE e $k$-CONJUNTO INDEPENDENTE pertençam à classe FPT quando parametrizados pelo tamanho da solução $k$, ambos não admitem núcleo polinomial. Primeiramente provamos que problema $k$-CLIQUE não admite núcleo polinomial através de uma PPT-redução do problema $k$-RAMSEY.

Teorema 3. $k$-CLIQUE quando o grafo de entrada é $G \bar{G}$ e o parâmetro é o tamanho da solução k não admite núcleo polinomial, a menos que $N P \subseteq \operatorname{coNP} /$ poly.

Mostramos que o problema dos conjuntos independentes em grafos prismas complementares também não admite núcleo polinomial (Corolário 6). Entretanto, diferentemente de cliques, os conjuntos independentes não são tão bem comportados nos prismas 
complementares. Sendo assim, dividiremos essa demonstração em duas etapas. Na primeira (Lema 4), é demonstrada uma PPT-redução do problema $k$-SPLIT para o problema $k$-Conjunto InDEPENDENTE. A segunda (Teorema 5) demonstrada uma PPT-redução do problema $k$-RAMSEY para o problema $k^{\prime}$-SPLIT onde $k^{\prime}=3 k$.

Lema 4. $k$-SPLIT quando parametrizado pelo tamanho da solução $k$ é PPT-redutível ao problema $k^{\prime}$-CONJUNTO INDEPENDENTE tendo como grafo de entrada um prisma complementar $G \bar{G}$ e parametrizado pelo tamanho da solução $k^{\prime}=k$.

A redução do problema $k$-RAMSEY para o problema $k$-SPLIT é formalmente apresentada a seguir:

Teorema 5. $k$-RAMSEY parametrizado pelo tamanho da solução $k$ é PPT-redutível ao $k^{\prime}$-SPLIT parametrizado pelo tamanho da solução $k^{\prime}=3 k$.

Transitivamente a partir do Lema 4 e do Teorema 5 temos que $k$-CONJUNTO INDEPENDENTE não admite núcleo polinomial.

Corolário 6. $k$-CONJUNTO INDEPENDENTE quando o grafo de entrada é $G \bar{G}$ e o parâmetro é o tamanho da solução $k$ não admite núcleo polinomial, a menos que $N P \subseteq \operatorname{coNP} /$ poly.

\section{Conclusões e Considerações Finais}

Neste trabalho, estudamos o problema de $k$-CLIQUE e $k$-CONJUNTO INDEPENDENTE tendo como entrada a classe de grafos prisma complementar. Utilizando como ferramenta a Teoria de Ramsey, provamos que tanto o problema $k$-CLIQUE quanto o problema $k$ CONJUNTO INDEPENDENTE em grafos prismas complementares pertencem à classe FPT quando parametrizados pelo tamanho da solução $k$, ou seja, são Tratáveis por Parâmetro Fixo. Além disso, através de PPT-reduções mostramos que ambos os problemas não admitem núcleo polinomial. Finalmente, é interessante evidenciar que a inviabilidade de núcleo polinomial para o problema $k$-CONJUNTO INDEPENDENTE quando parametrizado pelo tamanho da solução $k$ foi obtida de forma transitiva a partir do problema intermediário $k$-SPLIT. Sendo assim, o problema de determinar se um grafo possui um subgrafo split de cardinalidade $k$ é FPT, e a menos que $N P \subseteq c o N P / p o l y$, não admite núcleo polinomial.

\section{Referências}

Bondy, J. A. and Murty, U. S. R. (1976). Graph theory with applications, volume 290. Macmilan.

Cygan, M., Fomin, F. V., Kowalik, Ł., Lokshtanov, D., Marx, D., Pilipczuk, M., Pilipczuk, M., and Saurabh, S. (2015). Parameterized algorithms, volume 3. Springer.

dos Santos, V. F. and dos Santos Souza, U. (2015). Uma introdução à complexidade parametrizada.

Duarte, M. A., Penso, L., Rautenbach, D., and dos Santos Souza, U. (2017). Complexity properties of complementary prisms. Journal of Combinatorial Optimization, 33(2):365-372.

Erdös, P. and Szekeres, G. (1935). A combinatorial problem in geometry. Compositio Mathematica, 2:463-470.

Haynes, T. W., Henning, M. A., Slater, P. J., and van der Merwe, L. C. (2007). The complementary product of two graphs. Bulletin of the Institute of Combinatorics and its Applications, 51:21-30. 